
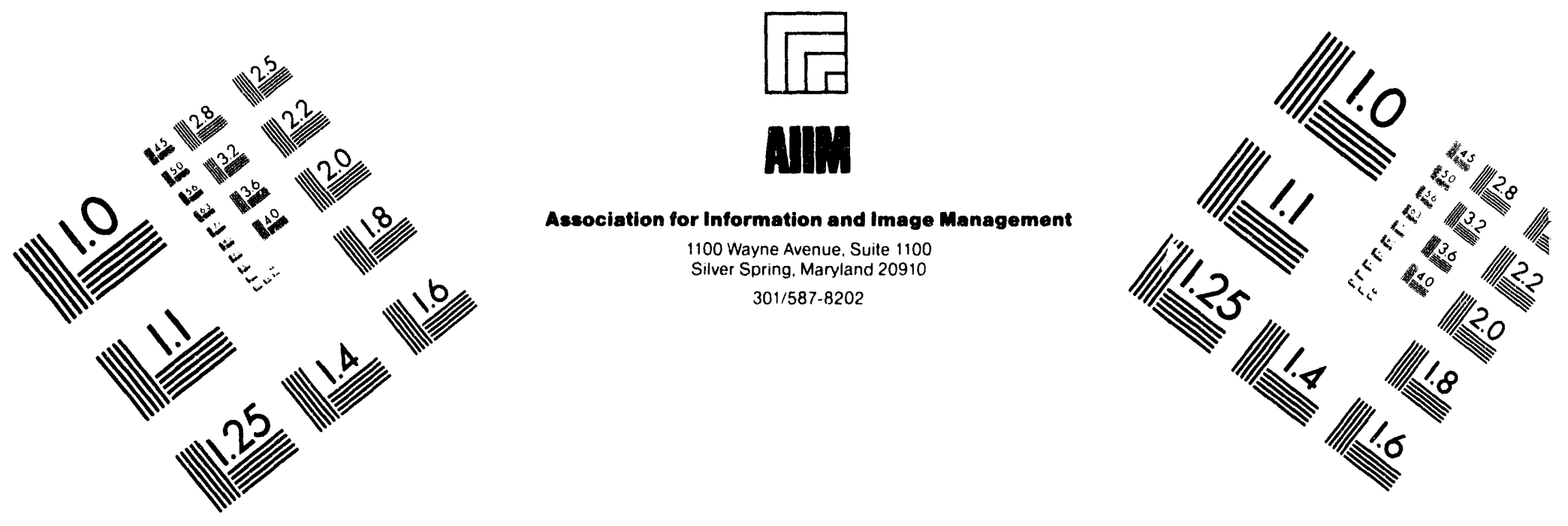

\title{
Centimeter
}

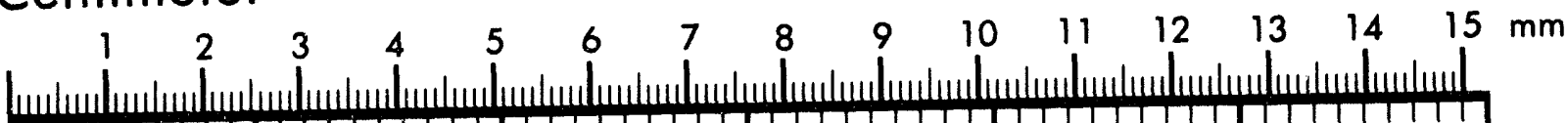

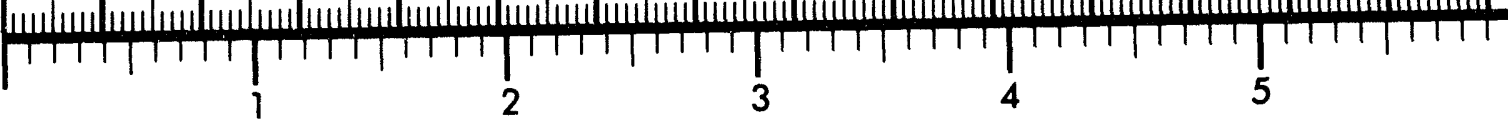
Inches
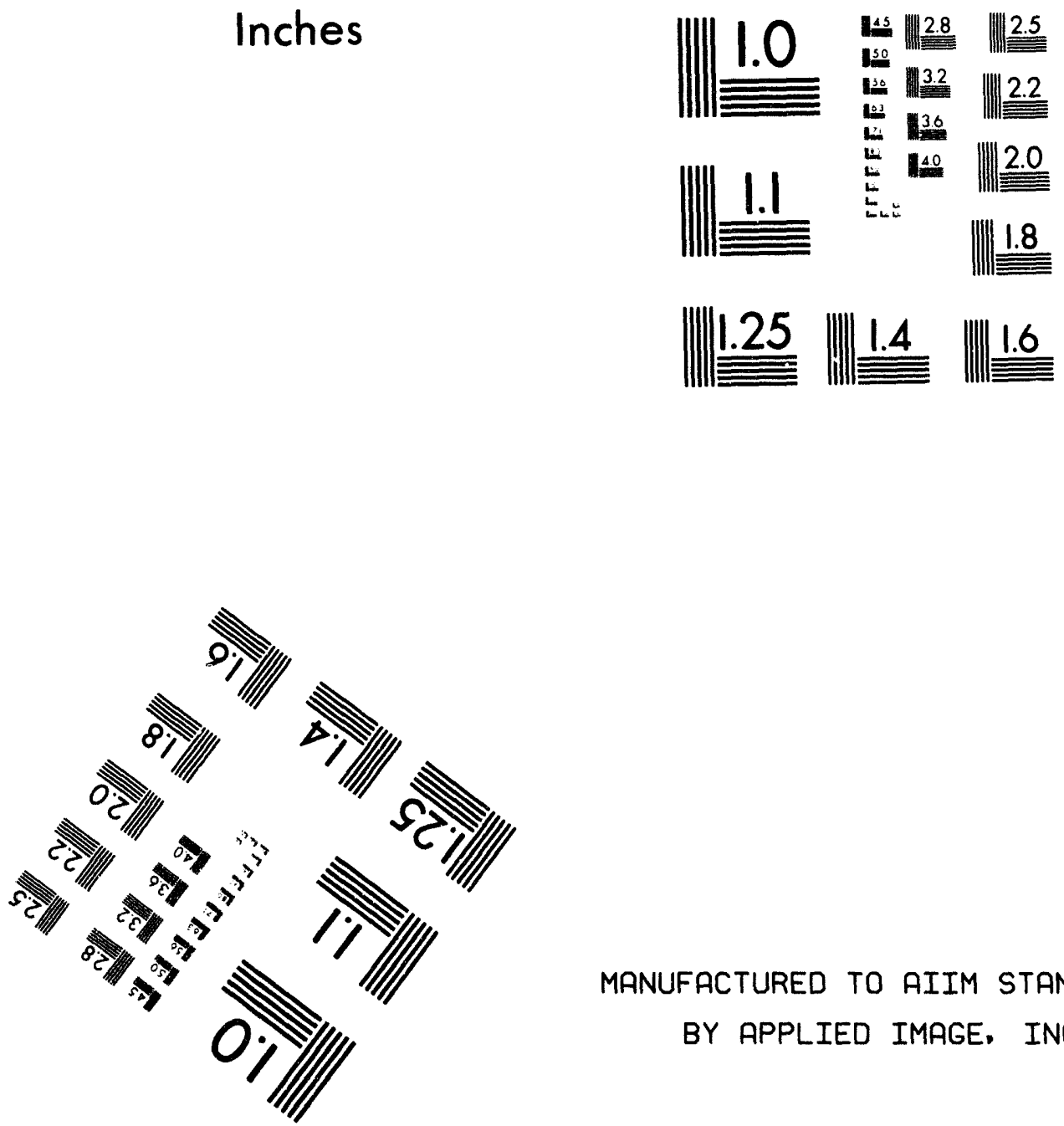

MANUFACTURED TO AIIM STANDARDS

BY APPLIED IMAGE, INC.

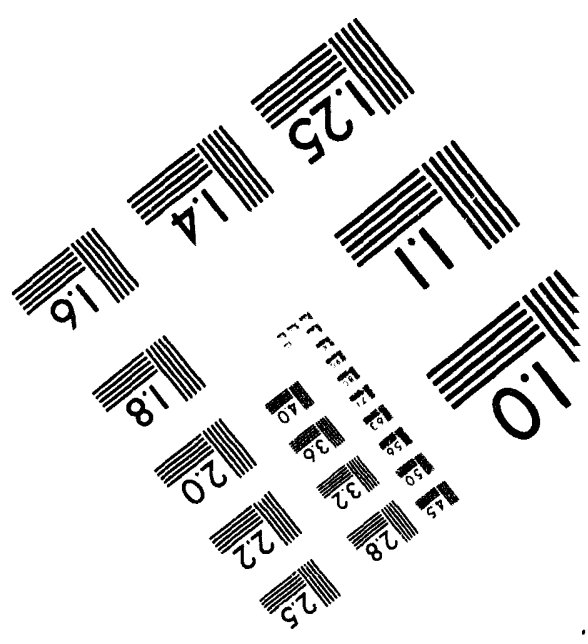



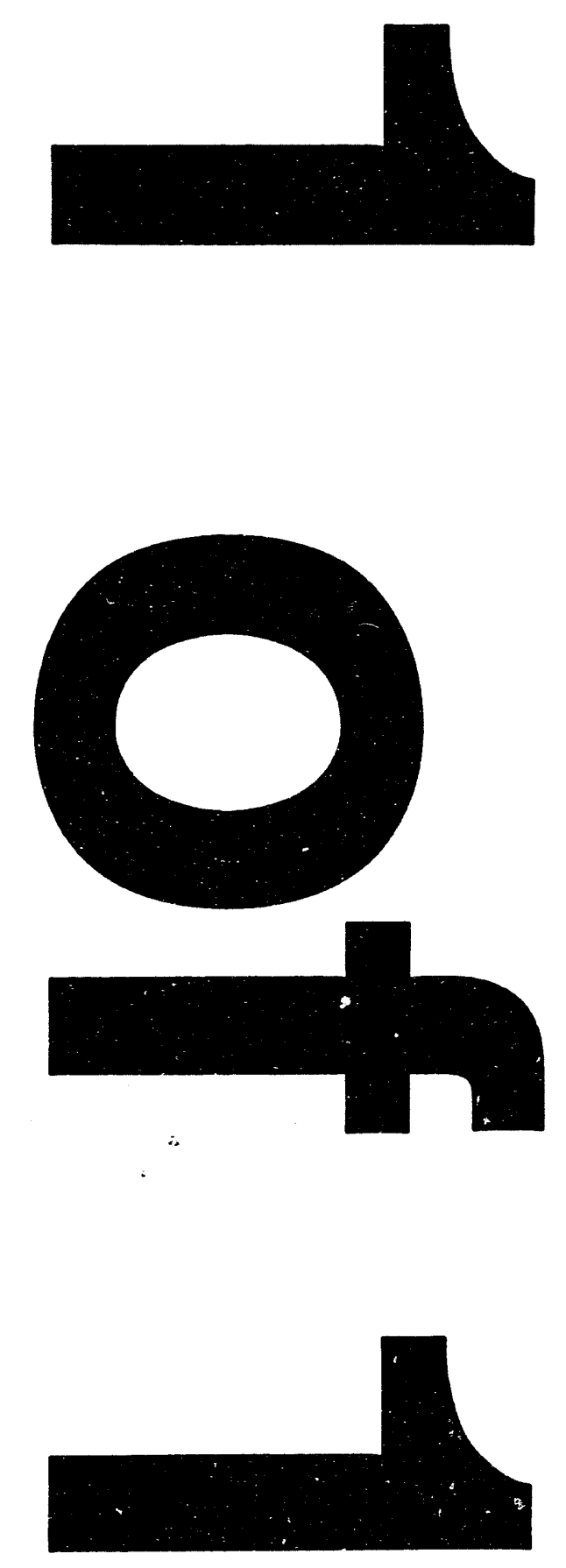


\section{Giant Magnetoresistance Calculated From First Principles}

W. H. Butler* , James M. MacLaren ** and X.-G Zhang ${ }^{\dagger}$

*Metals and Ceramics Division, Oak Ridge National Laboratory, Oak Ridge, TN 37831-6114

*-Department of Physics, Tulane University, New Orleans, Louisiana 70118

tCenter for Computational Sciences, University of Kentucky, Lexington, Kentucky 40506-

0045

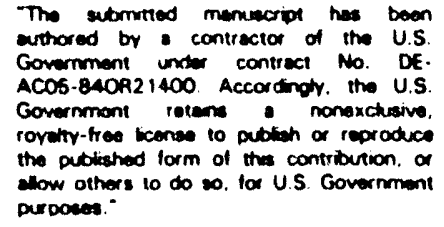

ABSTRACT

The Layer Korringa Kohn Rostoker-Coherent Potential Approximation technique was used to calculate the low temperature $\mathrm{Co} \mid \mathrm{Cu}$ and permalloy $\mid \mathrm{Cu}$ superlattices. Our calculations predict large giant magnetoresistance ratios for $\mathrm{Co} \mid \mathrm{Cu}$ and extremely large ratios for permalloy $\mid \mathrm{Cu}$ for current perpendicular to the layers. Mechanisms such as spin-orbit coupling which mix spin channels are expected to greatly reduce the GMR effect for permalloy $\mid \mathrm{Cu}$.

\section{Introduction}

An unexpectedly large effect of an applied magnetic field on the electrical resistance has been observed in a number of multilayer systems involving transition metals. The effect consists of a dramatic decrease in the electrical resistance in the presence of an applied magnetic field and has been observed in $\mathrm{Fe}$ - $\mathrm{Cr}$ multi-layers $[1,2,3,4,5,6]$, in $\mathrm{Co}-\mathrm{Cu}$ multilayers[3, 4,7$]$, in Co-Ru multilayers[3], and in bi-layers of $\mathrm{Ni} .8 \mathrm{Fe} .2$ separated by a thin layer of $\mathrm{Cu}[7,8]$. The effect is seen in systems in which alternate layers of magnetic atoms are aligned anti-parallel with one another in the absence of the applied field. Application of the magnetic field brings the layers into alignment and causes a decrease in the electrical resistivity both parallel to the layers and perpendicular to them.

The resistances of bcc $(100) \mathrm{Fe} /(100) \mathrm{Cr}[1]$ and fcc $(111) \mathrm{Co}(111) \mathrm{Cu}[4]$ have been observed to decrease by almost a factor of two when an applied magnetic field brings the magnetic moments on the layers into alignment. In this paper we present calculations of this giant magnetoresistance effect directly from first principles.

\section{Previous Theory}

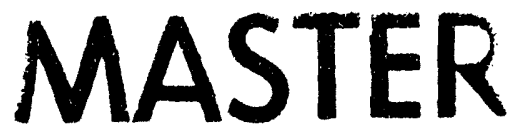

Baibich et. al.[1] who first reported the effect based on their observations on $\mathrm{Fe}-\mathrm{Cr}$ multi-layer systems observed that dilute $\mathrm{Cr}$ impurities in bulk Fe scatter majority spin electrons much more strongly than minority spin electrons. They proposed that the $\mathrm{Cr}$ atoms in the spacer layers might act the same way leading to a low resistance for the down spin electrons when the layers are aligned in parallel. The anti-parallel alignment would, in this picture, lead to a higher resistance by mixing the spin channels. Binasch et. al.[2] described the effect in terms of the necessity for electrons to undergo spin-flip scattering when traveling from one Fe layer into an adjacent one when the layers were aligned anti-parallel.

Camely and Barnaś and Barnaś et. al.[9, 10] generalized the semi-classical Boltzmann theory approach of Fuchs[11] and Sondheimer[12] to treat layered systems with different scattering properties in the two spin channels. Levy et. al.[13] and Zhang and Levy[14] developed a quantum mechanical treatment for spin dependent scattering in multilayer 
systems including both interfacial and bulk scattering. Both of these treatments utilize numerous adjustable parameters to account for the microscopic properties of these systems.

\section{Calculation Technique}

We have calculated the electronic structure of superlattices of copper and cobalt and of permalloy ( $\mathrm{Ni} .8 \mathrm{Fe} .2$ ) and $\mathrm{Cu}$ using the layer Korringa-Kohn-Rostoker technique[15] (LKKR) to solve the equations of density functional theory in the local spin density approximation $[16,17]$. The LKKR technique is particularly well adapted for calculating the properties of layered materials. It has the advantage over most other electronic structure techniques that the time required for a calculation scales linearly with the number of layers rather than as the third power of the number of layers. The LKKR also does not require that one treat all structures as three dimensional superlattices. The properties of isolated interfaces, for example, can be calculated directly. In the work described here, however, we have modeled the systems as superlattices because this simplified the application of transport theory.

The systems which we have chosen to model in these initial calculations consist of (111) superlattices of copper and sf either cobalt or permalloy. For the $\mathrm{Co} / \mathrm{Cu}$ superlattices, scattering arises from the interfacial layers which we have assumed to be $1 \%$ interdiffused; i.e. the interfacial $\mathrm{Cu}$ layers are assumed to have $1 \%$ of the copper atoms replaced by cobalt atoms which are randomly distributed within the layer. Similarly the interfacial cobalt layers are assumed to have $1 \%$ of their atoms randomly replaced by copper atoms. For the permalloy $\mid \mathrm{Cu}$ superlattices, scattering arises from the permalloy layers which are assumed to be random solid solution alloys.

The disorder is treated in the coherent potential approximation $[18,19]$ which provides a means of constructing an average Green function for a disordered solid solution alloy and which has been successfully applied to the calculation of many properties of alloys[20]. A novel feature of the LKKR is the ability it provides for allowing the concentration of each species to vary from layer to layer.

The calculation of the electrical resistivity is based on the use of a CPA-like approximation to evaluate the product of two Green functions which appears in the Kubo-Greenwood expression for the conductivity[21]. For these initial calculations of the GMR it is convenient to make two approximations in addition to those implicit in the KKR-CPA theory of resistivity described in reference[21]. Firstly, we assume that the energy bands are reasonably well defined, i.e. that the mean free path is significantly greater than a typical interatomic spacing. This assumption allows the KKR-CPA theory of transport to be reduced to a Boltzmann equation in which the the scattering probabilities are determined from first principles. The validity of this approximation can be checked since we calculate the mean free path for each point on the Fermi surface.

The second additional approximation that we have made in these calculations is the neglect of vertex corrections to the two particle propagator. This is equivalent to the neglect of the "scattering-in" terms in solving the Boltzmann equation. The regime of validity of this approximation is not quite so evident. It can be shown[22] that vertex corrections vanish if the individual scattering events are isotropic, or more generally, if they only connect states of the same parity, e.g. $s \rightarrow s, s \rightarrow d$, or $p \rightarrow p$. If the scattering is primarily in the forward direction, e.g. from a weak relatively long ranged potential (compared with the electron wavelength) the vertex corrections can be significant[23].

With these approximations the conductivity for spin $i$ in the $z$-direction in response to an electric field in the $z$-direction can be calculated as a sum over the states, $k$, of the 
superlattice,

$$
\sigma_{z z}^{i}=\frac{e^{2}}{V} \sum_{k} v_{z}^{i}(k) v_{z}^{i}(k) \tau^{i}(k)\left(-\frac{\partial f_{\mu}(E)}{\partial E}\right) .
$$

Here $V$ is the volume of the superlattice cell, $v_{z}^{i}=\hbar^{-1} \nabla_{k} E^{i}(k)$ is the electron group velocity, $\tau^{i}(k)$ is the Boltzmann lifetime defined below, and $f_{\mu}(E)$ is the Fermi function. At reasonable temperatures $\left(-\frac{\partial f_{\mu}(E)}{\partial E}\right)$ is effectively a delta function at the Fermi energy and the conductivity can be written as

$$
\sigma_{z z}^{i}=\frac{e^{2}}{\hbar(2 \pi)^{3}} \int_{\mu} \frac{d S_{k}}{\left|v^{i}(k)\right|} v_{z}^{i}(k) v_{z}^{i}(k) \tau^{i}(k)
$$

Using the fact that

$$
\frac{d S_{k}}{\left|v^{i}(k)\right|} v_{z}^{i}(k)=d k_{x} d k_{y}
$$

and recognizing that $v_{z}^{i}(k) \tau^{i}(k)$ is simply the mean free path in the $z$-direction, $l_{z}^{i}$, we have,

$$
\sigma_{z z}^{i}=\frac{e^{2}}{\hbar(2 \pi)^{3}} \int d k_{x} d k_{y} l_{z}^{i}
$$

where the $z$-component of the mean free path, $l_{z}^{i}\left(k_{x}, k_{y}\right)$ is determine $\dot{d}$ by the imaginary part of the solution of the secular equation. Thus $l\left(k_{x}, k_{y}\right)=1 /\left(2 \operatorname{Im} k_{z}\right)$ where $k_{z}$ is the (generally complex) solution to

$$
E\left(k_{x}, k_{y}, k_{z}\right)=\mu
$$

for given $k_{x}$ and $k_{y}$. The detailed justification for this identification of the mean free path or the lifetime with the complex dispersion relation within KKR-CPA theory is given in reference[21] and was used to successfully calculate the electrical resistivity of $\mathrm{Ag}$ - $\mathrm{Pd}$ and other alloys[22].

Finally, in the calculations presented here, we neglect mechanisms that would mix the majority and minority scattering channels. Examples of such mechanisms which would be effective at zero temperature are spin-orbit coupling and any misalignment in the orientation of the moments of the impurities. At higher temperature, spin waves would be effective in mixing the scattering channels.

\section{Results for $\mathrm{Cu} \mid \mathrm{Co}$}

Table I gives the calculated conductivities, lifetimes, and resistivities for multilayers with equal thicknesses of $\mathrm{Cu}$ and $\mathrm{Co}$. The quoted conductivities and resistivities are for a single spin channel. The $\uparrow$ symbol indicates the majority spin channel, $\downarrow$ denotes the minority spin channel, and $A$ indicates either of the spin channel, for the case in which the ferromagnetic layers alternate in moment direction (anti-ferromagnetic alignment). The calculated mean free path $\left\langle l_{z}^{i}\right\rangle$ is the average of the component of the mean free path in the $z$-direction defined as

$$
<l_{z}^{i}>=\frac{\int d k_{x} d k_{y} \sum_{n} l_{z}^{\text {in }}}{\int d k_{x} d k_{y}}
$$

where the sum over $n$ indicates that more than one sheet of the Fermi surface may have a projection on a given point in the two dimensional zone. This definition of an average mean free path may differ from other definitions and the appropriate definition for use in comparison to experiments on realistic systems may depend on the experiment and on the details of the Fermi surface. 
Table I: Calculated mean free paths, single channel resistivities, parallel and anti-parallel resistivities and GMR ratios for $\mathrm{Cu}$ /Co superlattices. Currents are assumed to be perpendicular to the layers. $\mathrm{Cu} 6 \mathrm{Co} 6$, for example, is a superlattice with 6 layers of $\mathrm{Cu}$ alternating with 6 layers of Co.

\begin{tabular}{ccccccccc}
\hline system & $\left\langle l_{z}^{\top}\right\rangle$ & $\left\langle l_{z}^{!}\right\rangle$ & $\left\langle l_{z}^{A P}\right\rangle$ & $\rho^{\top}$ & $\rho^{\top}$ & $\rho^{A P}$ & $\rho^{P}$ & $\rho^{A P} / \rho^{P}$ \\
\hline units & $\mathrm{nm}$ & $\mathrm{nm}$ & $\mathrm{nm}$ & $\mu \Omega \mathrm{cm}$ & $\mu \Omega \mathrm{cm}$ & $\mu \Omega \mathrm{cm}$ & $\mu \Omega \mathrm{cm}$ & \\
\hline $\mathrm{Cu} 1 \mathrm{Co} 1$ & 9353 & 280 & 334 & 0.588 & 19.66 & 8.24 & 0.639 & 12.90 \\
$\mathrm{Cu} 2 \mathrm{Co} 2$ & 6341 & 199 & 310 & 0.867 & 27.59 & 8.86 & 0.903 & 9.80 \\
$\mathrm{Cu} 3 \mathrm{Co} 3$ & 14279 & 221 & 377 & 0.385 & 24.87 & 7.30 & 0.425 & 17.17 \\
$\mathrm{Cu} 4 \mathrm{Co} 4$ & 16259 & 228 & 341 & 0.338 & 24.12 & 8.06 & 0.379 & 21.23 \\
$\mathrm{Cu} 5 \mathrm{Co} 5$ & 15014 & 356 & 480 & 0.366 & 15.45 & 5.72 & 0.431 & 13.28 \\
$\mathrm{Cu} 6 \mathrm{Co} 6$ & 32754 & 469 & 633 & 0.168 & 11.73 & 4.34 & 0.253 & 17.14
\end{tabular}

The column labeled $\rho^{A P}$ is the calculated resistivity for anti-parallel arrangement of the magnetic moments in neighboring Co layers for both spin channels. The resistivity for the two spin channels is identical in this case so the total resistance for both spin channels will be half of the single channel value. The column labeled $\rho^{P}$ is the net resistance (both channels) when the Co moments on all layers are parallel.

The magnitudes of the resistances depend on the amount of assumed interfacial interdiffusion, but we expect that the GMR ratio would not be affected if the amount of interdiffusion were increased under the assumption used here that all of the moments are aligned. The magnitude of the GMR effect would be reduced by scattering mechanisms such as phonons, vacancies, or grain boundaries that scatter the majority and minority spins more democratically and it would be greatly reduced by scattering mechanisms such as misaligned moments, e.g. in the interfacial regions, that cause a mixing of the spin channels and by spin waves at higher temperatures.

For reference, the calculated single channel resistivities (in $\mu \Omega \mathrm{cm}$ ) of the dilute bulk alloys were $\mathrm{Cu}(1 \% \mathrm{Co}): \rho^{\uparrow}=1.6, \rho^{\downarrow}=19.2$ and $\mathrm{Co}(1 \% \mathrm{Cu}): \rho^{\uparrow}=0.42, \rho^{\downarrow}=4.92$. These are in reasonable agreement with the experimental values of the total resistance of 5.8 for $\mathrm{Cu}(\mathrm{Co})[24]$ and 0.89 for $\mathrm{Co}(\mathrm{Cu})[25]$ if it is remembered that the interpretation of the resistance of dilute $\mathrm{Co}$ in $\mathrm{Cu}$ is complicated by spin orientation and Kondo effects.

\section{Results for Permalloy|Cu}

Table II gives the calculated results for permalloy $\mid \mathrm{Cu}$ superlattices for equal thicknesses of the Ni.8Fe.2 layers and the copper layers. The calculations are for currents perpendicular to the layers. The rernarkable feature of the results is that the calculated mean free paths are extremely long for the majority channel and extremely short for the minority channel. This leads to extremely large values of the predicted GMR ratio. Values of the resistivity in excess of $200 \mu \Omega \mathrm{cm}$ in the minority channel are unrealistic and result from a breakdown in the assumption that the bands are well defined. However, very strong scattering and high resistivities (on the order of $100 \mu \Omega \mathrm{cm}$ ) in the down spin channel should be expected since the potentials of $\mathrm{Cu}, \mathrm{Ni}$, and $\mathrm{Fe}$ are very different for minority electrons.

The long mean free paths and low resistivities calculated for the majority electrons result from the fact that the calculated majority $\mathrm{Ni}$ and Fe potentials have almost identical scattering properties at the Fermi energy. The scattering properties of the $\mathrm{Cu}$ atoms are almost as close but do not match quite as well. This should not be surprising since $\mathrm{Cu}$ has 11 , valence electrons per atom, $\mathrm{Ni}$ has 10 and $\mathrm{Fe}$ has 9 . Since Fe forms a moment of about $2.6 \mu_{B}$ and $\mathrm{Ni}$ a moment of about $0.6 \mu_{B}$, the number of electrons per atom for the 
Table II: Calculated mean free paths, single channel resistivities, parallel and anti-parallel resistivities and GMR ratios for permalloy $(\mathrm{P}) \mid \mathrm{Cu}$ superlattices. Currents are assumed to be perpendicular to the layers.

\begin{tabular}{ccccccccc}
\hline system & $\left\langle l_{z}^{\dagger}\right\rangle$ & $\left\langle l_{z}^{!}\right\rangle$ & $\left\langle l_{z}^{A P}\right\rangle$ & $\rho^{\top}$ & $\rho^{\perp}$ & $\rho^{A P}$ & $\rho^{P}$ & $\rho^{A P} / \rho^{P}$ \\
\hline units & $\mathrm{nm}$ & $\mathrm{nm}$ & $\mathrm{nm}$ & $\mu \Omega \mathrm{cm}$ & $\mu \Omega \mathrm{cm}$ & $\mu \Omega \mathrm{cm}$ & $\mu \Omega \mathrm{cm}$ & \\
\hline P1Cu1 & 707 & 0.782 & 1.291 & 0.202 & 183 & 55.5 & 0.202 & 274 \\
P2Cu2 & 681 & 1.118 & 1.668 & 0.210 & 128 & 43.0 & 0.210 & 204 \\
P3Cu3 & 662 & 0.557 & 1.062 & 0.216 & 256 & 67.5 & 0.216 & 312 \\
P4Cu4 & 528 & 0.286 & 0.540 & 0.271 & 500 & 132.5 & 0.271 & 489 \\
P5Cu5 & 623 & 0.191 & 0.468 & 0.230 & 750 & 152.5 & 0.230 & 664 \\
P6Cu6 & 794 & 0.119 & 0.243 & 0.180 & 1206 & 294.1 & 0.180 & 1633
\end{tabular}

majority spins is 5.5 for $\mathrm{Cu}, 5.3$ for $\mathrm{Ni}$, and 5.3 for Fe. According to our calculations the difference in the number of electrons per atom in the self-consistent majority potentials for $\mathrm{Ni}$ and $\mathrm{Fe}$ is approximately 0.03 . In contrast, the numbers of minority electrons per atom on the $\mathrm{Cu}, \mathrm{Ni}$, and $\mathrm{Fe}$ atoms is approximately, 5.5, 4.7, and 3.2. It should be remembered that the scattering rates scale as the square of the potential difference.

It is clear, however, that the calculation omits a scattering mechanism that significantly affects the majority electrons. The calculated resistivity of bulk permalloy is $0 \cap 38 \mu \Omega \mathrm{cm}$, approximately 100 times smaller than the experimental value[26]. It is interesting to note that Mertig et. al. [27] obtained a calculated resistivity that is also two orders of magnitucde too small in a very thorough study of the single impurity limit for $\mathrm{Fe}$ in $\mathrm{Ni}$. The resistance of very dilute alloys of $\mathrm{Fe}$ in $\mathrm{Ni}$ is reported to be[28] $0.39 \mu \Omega \mathrm{cm} / \mathrm{at}$.\%. If these calculations are correct, the implication is that most of the resistance in $\mathrm{Ni}(\mathrm{Fe})$ alloys is due to mechanisms outside the standard model. One obvious omission is the neglect of spin-orbit coupling.

\section{ACKNOWLEDGEMENTS}

It is a pleasure to acknowledge helpful discussions with V. S. Speriosu, B. A. Gurney, S. S. P. Parkin, R. K. Nesbet, B. A. Jones, P. A. Schroeder, D. M. Nicholson, and G. M. Stocks. Work at Oak Ridge was supported by the ORNL Exploratory Studies Program; work at Kentucky was supported by the High Performance Computing and Communication Program, U. S. Department of Energy under Contract No. DE-AC05-84OR21400 with Martin Marietta Energy Systems, Inc.

\section{REFERENCES}

1. M. N. Baibich, J. M. Broto, A. Fert, F. N. Van Dau, and F. Petroff, Phys. Rev. Lett. 61, 2472 (1988).

2. G. Binasch, P. Grünberg, F. Saurenbach, and W. Zinn, Phys. Rev. 39, 4828 (1989).

3. S. S. P. Parkin, N. More, K. P. Roche, Phys. Rev. Lett. 64, 2304 (1990).

4. S. S. P. Parkin, R. Bhadra, and K. P. Roche, Phys. Rev. Lett. 66, 2152 (1991).

5. A. Chaiken, T. M. Tritt, D. J. Gillespie, J. J. Krebs, P. Lubitz, J. Appl. Phys. 69, 4798 (1991). 
6. P. Grünberg, S. Demokritov, A. Fuss, M. Vohl, and J. A. Wolf, J. Appl. Phys. 69, 4789 (1991).

7. B. Dieny, V. S. Speriosu,S. Metin, S. S. P. Parkin, B. A. Gurney, P. Baumgart, and D. R. Wilhoit, J. Appl. Phys. 69, 4774 (1991).

8. B. Dieny, V. S. Speriosu, S. S. P. Parkin, B. A. Gurney, D. R. Wilhoit, and D. Mauri, Phys. Rev. B 43, 1297 (1991).

9. R. E. Camely, and J. Barnaś, Phys. Rev. Lett. 63, 664 (1989).

10. J. Barnaś, A. Fuss, R. E. Camley, P. Grünberg, and W. Zinn. Phys. Rev. B 42, 8110 (1990).

11. K. Fuchs, Proc. Cambridge Philos. Soc. 34, 100 (1938).

12. E. H. Sondheimer, Adv. Phys. 1, 1 (1952).

13. P. M. Levy, S. Zhang, and A. Fert, Phys. Rev. Lett. 65, 1643 (1990).

14. S. Zhang, P. M. Levy, J. Appl. Phys. 69, 4786 (1991).

15. J. M. MacLaren, S. Crampin, D. D. Vvedensky and J. B. Pendry, Phy's. Rev. B 40, 12164 (1989).

16. P. Hohenberg and W. Kohn, Phys. Rev. 136 B, 864 (1964).

17. W. Kohn and L. J. Sham, Phys. Rev. B 140, A1133 (1965).

18. P. Soven, Phys. Rev. 156, 809 (1967).

19. D. W. Taylor, Phys. Rev. 1561017 (1967).

20. J. S. Faulkner, Prog. Mat. Sci. 27, 1 (1982).

21. W. H. Butler, Phys. Rev. B, 31, 3260 (1985).

22. W.H. Butler and G. M. Stocks, Phys. Rev. B, 29, 4217 (1984).

23. J. C. Swihart, W. H. Butler, G. M. Stocks, D. M. Nicholson, and R. C. Ward, Phys. Rev. Lett. 57, 1181 (1986).

24. G. J. van den Berg, J. van Hook, B. Knook, Proc. L.T. 10 4, 272 (1966).

25. J. Hugel, J. Phys. F 3, 1723 (1973).

26. J. Smit, Physica, 21, 877 (1955).

27. I. Mertig, R. Zeller, and P. H. Dederichs, in Application of Multiple Scattering Theory to Materials Science, edited by W. H. Butler, A. Gonis, P. H. Dederichs, and R. Weaver, MRS Symposia Proceedings No. 253 (Materials Research Society, Pittsburg, 1992)

28. T. Farrell and D. Greig, J. Phys. C: Solid St. Phys. 1, 1359 (1968). 


\section{0 $+66141 d \exists S$ व $\exists \wedge \exists \supset \exists \forall$}

\section{DISCLAIMER}

This report was prepared as an account of work sponsored by an agency of the United States Government. Neither the United States Government nor any agency thereof, nor any of their employees, makes any warranty, express or implied, or assumes any legal liability or responsibility for the accuracy, completeness, or usefulness of any information, apparatus, product, or process disclosed, or represents that its use would not infringe privately owned rights. Reference herein to any specific commercial product, process, or service by trade name, trademark, manufacturer, or otherwise does not necessarily constitute or imply its endorsement, recoinmendation, or favoring by the United States Government or any agency thereof. The views and opinions of authors expressed herein do not necessarily state or reflect those of the United States Government or any agency thereof. 

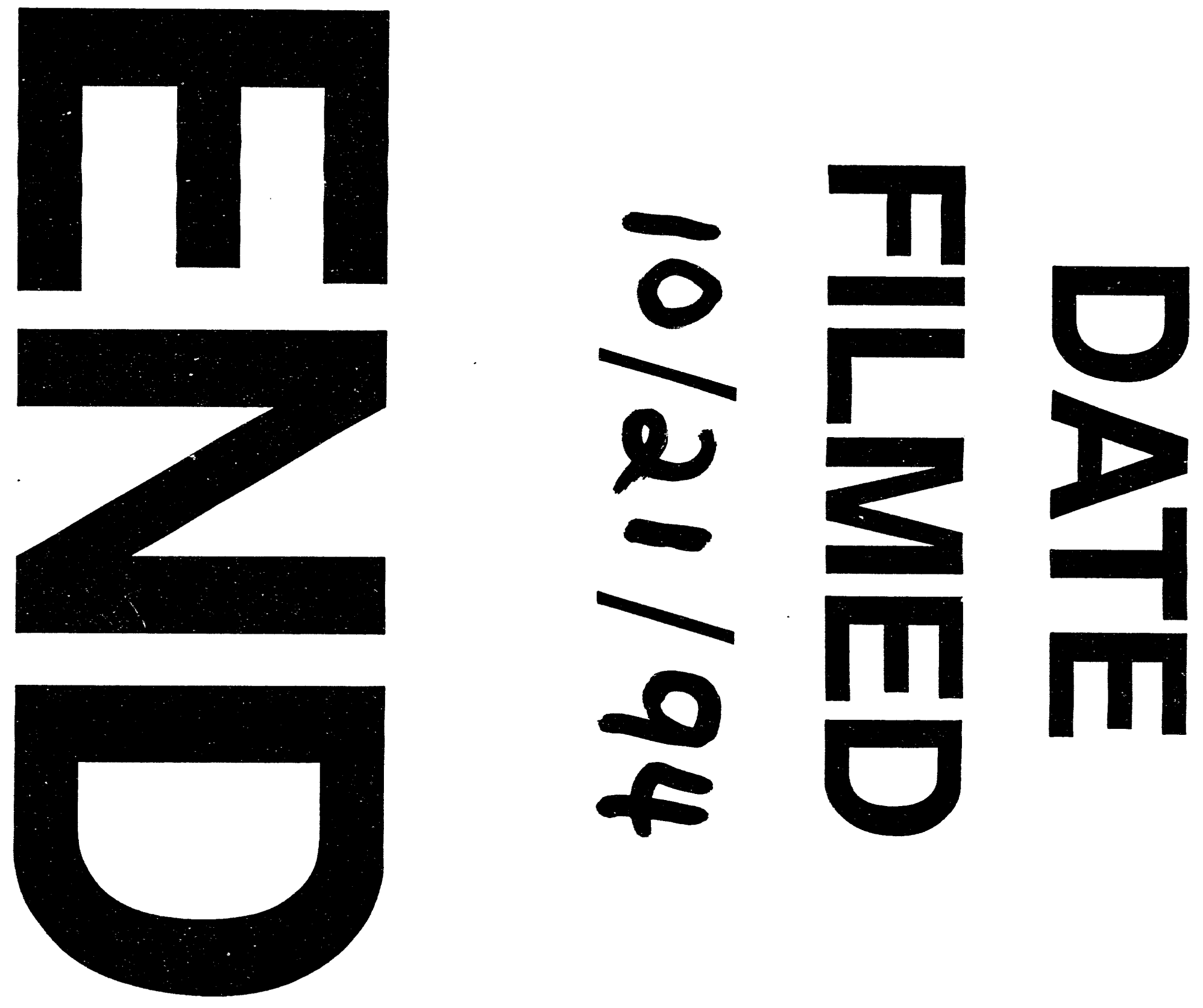
